STRUCTURAL SCIENCE CRYSTAL ENGINEERING MATERIALS

ISSN 2052-5206

\section{The mechanism of solvent-mediated desolvation transformation of lenvatinib mesylate from di- methyl sulfoxide solvate to form D}

\author{
Zhixin Zheng, ${ }^{a}$ Baohong Hou, ${ }^{a, b}$ Xiaowei Cheng, ${ }^{a}$ Wanying Liu, ${ }^{a}$ Xin Huang, ${ }^{a, b}$ \\ Ying Bao, ${ }^{\mathrm{a}, \mathrm{b}}$ Ting Wang, ${ }^{\mathrm{a}, \mathrm{b}}$ Zhao Wang ${ }^{\mathrm{a}, \mathrm{b}}$ and Hongxun $\mathrm{Hao}^{\mathrm{a}, \mathrm{b}}$ *
}

Received 16 January 2020

Accepted 18 March 2020

Edited by M. Du, Tianjin Normal University, People's Republic of China

Keywords: lenvatinib mesylate; solubility; solvent-mediated desolvation transformation; water activity; mechanism.

Supporting information: this article has supporting information at journals.iucr.org/b

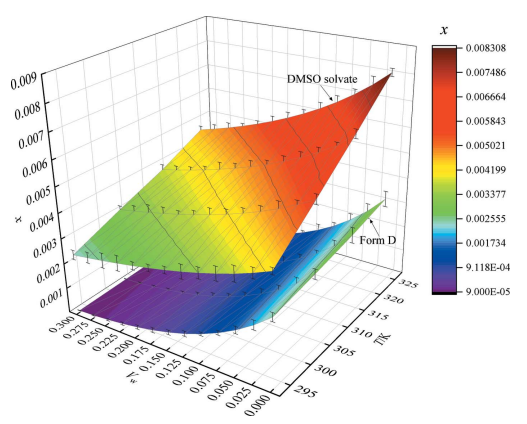

\section{Introduction}

Nowadays, screening new solid forms of an active pharmaceutical ingredient (API) is an indispensable step in the development and production of drugs. The physical properties of different solid forms of the same compound, such as melting point, solubility, dissolution rate, physical and chemical stability, etc., may vary significantly. These properties will further affect the bioavailability, fluidity and efficacy of the final products (Schöll et al., 2006; Llinàs \& Goodman, 2008; Bērziņš et al., 2017; Brittain, 2009; Bond, 2009). Different crystal forms of multifarious drugs have been screened by controlling various factors influencing the crystallization process, such as temperature, solvent, humidity, solid loading, stirring rate, additives and template materials (Jiang et al., 2015; Hao et al., 2010, 2012; Guo et al., 2018; Barbas et al., 2018; 
Shi et al., 2015; Zong et al., 2017; Yang et al., 2013, 2012; Ouyang et al., 2014). In fact, many substances have far more solvated forms than the unsolvated form. For example, methyl cholate has eight unsolvated forms and 27 solvates (Bērziņš et al., 2017). Sulfathiazole has more than 100 solvates but only five unsolvated forms (Anwar et al., 1989; Apperley et al., 1999; Bingham et al., 2001). Therefore, the study of solvates should be of great concern.

Solvates have a wide application prospect. Firstly, solvates can be used in the purification of substances. For example, form $\mathrm{R}_{1}$ of S-enzalutamide can be easily contaminated by its substitution impurity O-enzalutamide. However, the impurity can be removed by desolvating isopropanol solvate $\mathrm{R}_{3}$ which is formed by the solvation of form $\mathrm{R}_{1}$ (Maini et al., 2018). Dirithromycin and piperidene can also be purified from erythromycylamine and the ' $\mathrm{N}$ linked' impurity precursor of piperidene by their acetone solvates and hydrates, respectively (Wirth \& Stephenson, 1997; Black et al., 2004). Secondly, solvates having superior properties can be used in the form of a commercial product, such as the DMSO solvate of trametinib, the acetone solvates of cabazitaxel and ledipasvir, the ethyl acetate solvate of voxilaprevir, etc. Thirdly, new polymorphs can be obtained by desolvating specific types of solvates. For instance, form D of prilocaine hydrochloride can only be prepared by desolvating its dioxane solvate (Schmidt et al., 2004) and its new crystal form AH D can only be obtained by the dehydration of thymine hydrate (Braun et al., 2016). Therefore, it is necessary to carry out intensive investigations on the solvate formation and desolvation process.

Solvent-mediated solid phase transformation is one kind of transformation process from a metastable solid form to a more stable solid form in a solvent environment (Mangin et al., 2009), which includes the dissolution of metastable solid form and the nucleation and growth of a stable solid form (Gu et al., 2001; Jiang et al., 2010). The determination of the rate control step of the solvent-mediated solid phase transformation process is essential to explore the transformation mechanism and better control of the transformation process. The rate control steps in the transformation process are different for different APIs and solvent systems. According to the real-time relationship between the completion degree of solid phase and the concentration of solution in the solid phase transformation process, O'Mahony et al. (2012) summarized four diverse circumstances, namely dissolution-controlled, growth-

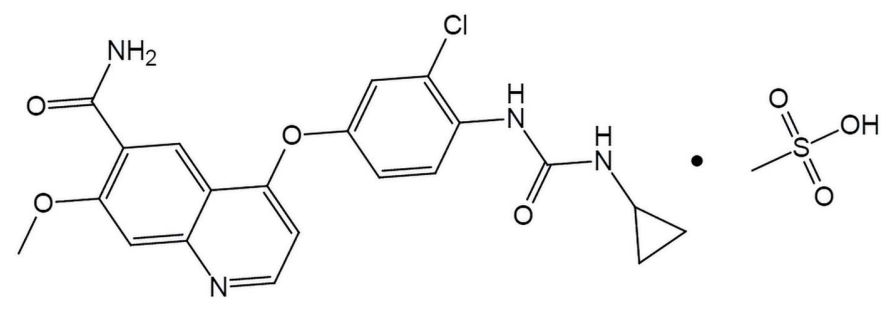

Figure 1

The molecular formula of lenvatinib mesylate, where the moculare formila on the left is molecular lenvatinib and the molecule on the right is a methansulfonic acid molecule.
Table 1

All the discovered solid forms of LM.

Forms as reported by Matsushima et al. (2005) and Sardone et al. (2018).

\begin{tabular}{ll}
\hline Nomenclature & Solid form \\
\hline Form A & Unsolvated form \\
Form B & Unsolvated form \\
Form C & Unsolvated form \\
Form F & Hydrate \\
Form I & Acetic acid solvate \\
Form DMSO-1 & Hydrate \\
Form DMSO-2 & Hydrate \\
Form ACA-1 & Acetic acid solvate \\
Form ACA-1 HT dry & Unsolvated form \\
Form $\mathrm{H}_{2} \mathrm{O}-1$ & Unsolvated form \\
Form COF-1 & Chloroform solvate \\
Form FOA-1 & Formic acid solvate \\
\hline
\end{tabular}

controlled, nucleation-dissolution-controlled and nucleationgrowth-controlled solid phase transformation processes.

The model compound lenvatinib mesylate (CAS registry NO: 857890-39-2, $\mathrm{C}_{22} \mathrm{H}_{23} \mathrm{ClN}_{4} \mathrm{O}_{7} \mathrm{~S}$; the chemical structure is shown in Fig. 1), 4-[3-chloro-4-( $N^{\prime}$-cyclopropylureido)phenoxy]-7-methoxyquinoline-6-carboxamide methane sulfonate (denoted as LM), is one kind of oral multikinase inhibitor. Being a great-promising anticancer drug, LM has passed phase 3 clinical trials for differentiated thyroid cancer and is undergoing phase 3 clinical trials for liver cancer (Okamoto $e t$ al., 2015). Until now, five unsolvated crystal forms, three hydrate crystal forms, two acetic acid solvates, one chloroform solvate, and one formic acid solvate of LM have been discovered (Matsushima et al., 2005; Sardone et al., 2018). All the discovered solid forms of lenvatinib mesylate are listed in Table 1. However, the reported forms are more or less flawed. Form B will slowly transform to form $\mathrm{C}$ under high humidity conditions while the solubility of stable form $\mathrm{C}$ is too low and hydrate form $\mathrm{F}$ will transform to form $\mathrm{C}$ in a solution system with a water activity ranging from 0 to 0.821 . Acetic acid solvate form I is not only hygroscopic but also unstable. Because of chloroform belonging to the second class of solvents which are restricted (FDA classification of solvents) in use and formic acid is strongly acidic, chloroform solvate CHF-1 and formic acid solvate FOA-1 are not applicable for pharmaceutical purposes. Acetic acid solvate ACA-1, anhydride ACA-1 HT dry and $\mathrm{H}_{2} \mathrm{O}-1$ are all thermodynamically unstable. Moreover, the transformation relationships of different forms were not well investigated or understood. Thus, it is necessary to further investigate the solid state forms, crystal structures and crystal transformation behaviors of LM, which is essential for pharmaceutical development.

In this study, a new DMSO solvate and a new unsolvated form (form D) of LM were discovered and characterized for the first time. The thermodynamic mechanism of the solventmediated desolvation transformation (SMDT) process from DMSO solvate to form D in DMSO-water mixed solvent was evaluated by measuring and analyzing their solubility data. The SMDT process was investigated in detail. The composi- 
tion of the solid phase was in situ monitored using Raman spectroscopy and the results were verified by quantitative powder X-ray diffraction. At the same time, the change of solute concentration in solution over time was determined through gravimetric analysis. Finally, the impacts of water activity and temperature on the SMDT process were investigated and discussed. A new SMDT mechanism was proposed based on the obtained results.

\section{Experimental}

\subsection{Materials}

The raw material of LM (form C) with a mass fraction purity higher than $99.0 \%$ was purchased from Jinan Sanzhi Pharmaceutical Technology Co., Ltd. DMSO and acetonitrile which are of analytical grade were supplied from Tianjin Kemiou Chemical Reagent Co., Ltd. Distilled water was prepared in our laboratory and used throughout all experiments.

\subsection{Preparation of two solid forms of $L M$}

LM DMSO solvate was prepared from LM form C through a cooling-antisolvent coupled crystallization method. LM form C $(1.00 \mathrm{~g})$ was dissolved in DMSO $(15 \mathrm{ml})$ under the stirring rate of $300 \mathrm{rpm}$ (controlled by Vertical Constant Speed Electric Mixer, HD2015W, Shanghai Sile Instrument Co., Ltd) at $343.15 \mathrm{~K}$ (controlled by Thermostatic Water Bath, XOYS2006N, Nanjing Xianou Instruments Manufacture Co., Ltd and measured by a mercury thermometer). After the solid was completely dissolved, acetonitrile $(50 \mathrm{ml})$ was added into the solution at a rate of $0.1 \mathrm{ml} \mathrm{min}^{-1}$ by a peristaltic pump (Dispensing Peristaltic Pump, BT100-1F, Baoding Longer Precision Pump Co., Ltd) while the temperature was decreased to $293.15 \mathrm{~K}$ at a rate of $0.1 \mathrm{~K} \mathrm{~min}^{-1}$ and then maintained at constant temperature of $293.15 \mathrm{~K}$ for $8 \mathrm{~h}$. Finally, the suspension was filtered and dried under vacuum at $313.15 \mathrm{~K}$ (Electrothermal Constant Temperature Drying Oven, DZ-2BCII, Tianjin Taisite Instrument Co., Ltd) to obtain LM DMSO solvate product.

LM form D was prepared from DMSO solvate by suspension transformation method. LM DMSO solvate $(1 \mathrm{~g})$ was added to a mixture of DMSO $(15 \mathrm{ml})$ and water $(5 \mathrm{ml})$ and stirred at $293.15 \mathrm{~K}$ with a stirring rate of $300 \mathrm{rpm}$ for $24 \mathrm{~h}$. After the transformation process was terminated, the suspension was filtered and dried under vacuum at $313.15 \mathrm{~K}$ to obtain LM form D product.

\subsection{Characterization methods}

Powder X-ray diffraction (PXRD) patterns of LM DMSO solvate and form $\mathrm{D}$ were obtained using a Rigaku D/max-2500 diffractometer in $2 \theta$ range from $2^{\circ}$ to $40^{\circ}$ with a scanning rate of $8^{\circ} \mathrm{min}^{-1}$, step size of $0.08^{\circ}$ and voltage of $40 \mathrm{kV}$ and current of $100 \mathrm{~mA}$.

A polarized light microscope (Series Biological Microscope, BK5000, Chongqing Optec Instrument Co., Ltd) was used to observe the exterior morphology of LM DMSO solvate and form D.

Thermogravimetric analysis (TGA) on a Mettler Toledo TASDT-Q600 instrument was used to determine the weight loss of LM DMSO solvate and form D. The temperature range was from 303.15 to $623.15 \mathrm{~K}$ with a heating rate of $5 \mathrm{~K} \mathrm{~min}^{-1}$ under a nitrogen atmosphere and the sample weight of 5$10 \mathrm{mg}$.

Differential scanning calorimetry (DSC) on a MettlerToledo DSC1 instrument was used to monitor the melting processes of LM DMSO solvate and form D. The samples of 5-10 mg were loaded into an aluminium crucible and scanned in the temperature range from 303.15 to $623.15 \mathrm{~K}$ with a heating rate of $5 \mathrm{~K} \mathrm{~min}^{-1}$ in a nitrogen atmosphere, so as to obtain the melting point and monitor the melting process of the two forms.

Raman spectra were collected with a Kaiser Raman RXN2 system (Kaiser Optical System, Inc., Ann Arbor, MI, USA). Solid powders were analyzed with a PhAT probe which was perpendicularly fixed above the solid powders at a distance of 2-3 mm. In transformation experiments, the immersion MR probe was inserted into the crystallizer and immersed into the suspension. Tin foil was used for shading during the whole experiment process. Raman spectra were collected in the range $100 \mathrm{~cm}^{-1}$ to $3200 \mathrm{~cm}^{-1}$ with a resolution of $0.5 \mathrm{~cm}^{-1}$ and an exposure time of $15 \mathrm{~s}$.

\subsection{The establishment of quantitative analysis method}

In order to confirm the mass fraction of the two crystal forms in the solid mixture, a PXRD calibration curve was established (Shi et al., 2018). It is well known that the intensity of the PXRD diffraction peaks of a crystal can be affected by a variety of factors, such as powder packing, sample thickness, crystallite size, type of sample holder and preferred orientation effects of the crystal (Li et al., 2011; Qiu et al., 2015; Croker et al., 2012). These factors were all taken into account when measuring the diffraction pattern of standard samples to minimize their influence on the result.

The standard samples were pure DMSO solvate, pure form $\mathrm{D}$ and mixtures of two forms with different proportions (mass fraction of DMSO solvate from 0.1 to 0.9, step size of 0.1). DMSO solvate and form $\mathrm{D}$ powder samples were ground individually in an agate mortar for $5 \mathrm{~min}$ and screened through a 400-mesh sieve to reduce the effect of crystal size on the preferred orientation. The standard samples were obtained by mixing the above powders of two forms in different proportions. Moreover, the sample stage and sample thickness were maintained at the same conditions to eliminate their influences on the final results during the PXRD diffraction pattern measurements. Meanwhile, all the standard samples were scanned from $2^{\circ}$ to $20^{\circ}$ on the $2 \theta$ scale with a scanning speed of $8^{\circ} \mathrm{min}^{-1}$ and a step size of $0.02^{\circ}$.

In the quantitative process, $2 \theta=6.9 \pm 0.2^{\circ}$ was selected as the characteristic peak of DMSO solvate while $2 \theta=12.3 \pm 0.2^{\circ}$ was selected as the characteristic peak of form D. Since the intensity of the characteristic peak of DMSO solvate and form 
Table 2

The values of parameters in the equation for calculating molar solubility of LM.

\begin{tabular}{llllll}
\hline & $\begin{array}{l}\rho_{\mathrm{d}} \\
\left(\mathrm{g} \mathrm{ml}^{-1}\right)\end{array}$ & $\begin{array}{l}\rho_{\mathrm{w}} \\
\left(\mathrm{g} \mathrm{ml}^{-1}\right)\end{array}$ & $\begin{array}{l}M_{\mathrm{d}} \\
\left(\mathrm{g} \mathrm{mol}^{-1}\right)\end{array}$ & $\begin{array}{l}M_{\mathrm{w}} \\
\left(\mathrm{g} \mathrm{mol}^{-1}\right)\end{array}$ & $\begin{array}{l}M_{\mathrm{LM}} \\
\left(\mathrm{g} \mathrm{mol}^{-1}\right)\end{array}$ \\
\hline $293.15 \mathrm{~K}$ & 1.100 & 0.998 & 78.13 & 18.02 & $\begin{array}{l}601.09 \\
\text { (DMSO solvate) }\end{array}$ \\
$303.15 \mathrm{~K}$ & & 0.996 & & & 522.96 \\
$323.15 \mathrm{~K}$ & & 0.992 & & & (Form D) \\
\hline
\end{tabular}

$\mathrm{D}$ are approximately equal under the same test conditions, the relative characteristic peak intensity of DMSO solvate can be selected to measure the mass fraction of DMSO solvate in the mixture, which can be calculated by equation (1):

$$
x_{\mathrm{Ds}}^{\prime}=\frac{I_{\mathrm{Ds}}}{I_{\mathrm{Ds}}+I_{\mathrm{D}}},
$$

where $x_{\mathrm{Ds}}^{\prime}$ is the calculated value of the mass fraction of DMSO solvate in the mixture, $I_{\mathrm{Ds}}$ and $I_{\mathrm{D}}$ represent the characteristic peak intensity of DMSO solvate and form D, respectively.

2.5. Solubility measurements and transformation equilibrium of two solid forms of LM

Solubility data of LM DMSO solvate and form D in mixed solvents of DMSO and water were measured from 293.15 to $323.15 \mathrm{~K}$. It was found that when the volume fraction of water in DMSO-water mixed solvent (denoted as $V_{\mathrm{w}}$ ) is higher than 0.300 , the viscosity of the system increased rapidly, resulting in the inability to obtain a clear saturated solution. Thus, only the solubility data with $V_{\mathrm{w}}$ ranging from 0 to 0.300 and a step of 0.025 was obtained. $V_{\mathrm{w}}=0.250$ was taken as an example to explain the solubility measurement process: Excess LM form D was dissolved in a mixed solvent of $15 \mathrm{ml}$ DMSO and $5 \mathrm{ml}$ water to saturate the solution. The suspension was stirred at a

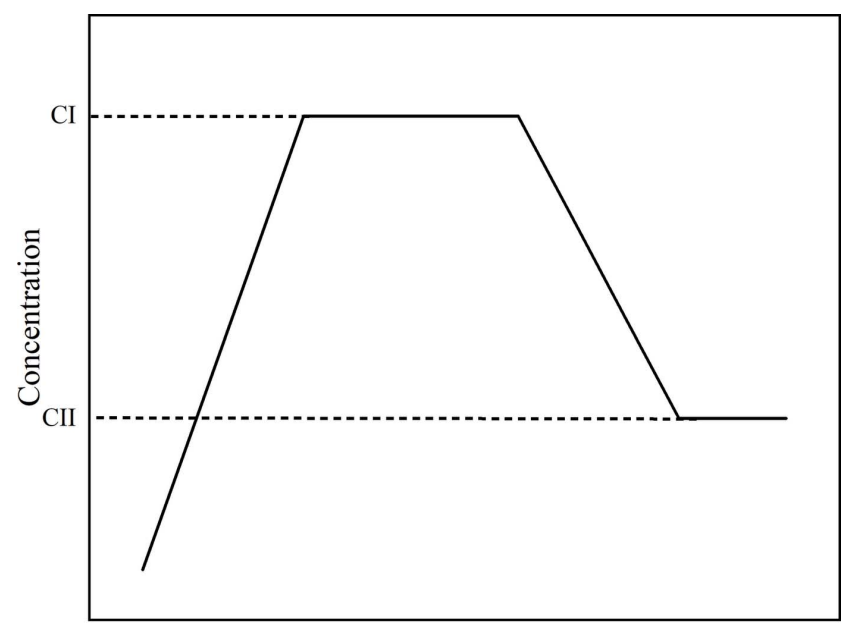

Figure 2

Time

Schematic diagram of the concentration of solution as a function of time, which displays the process of transformation from metastable form to stable form. CI is the solubility of the metastable form and CII is the solubility of the stable form. stirring rate of $300 \mathrm{rpm}$ for $24 \mathrm{~h}$ and the temperature was controlled by a thermostatic water bath. Then, the suspension was filtered and the PXRD of the undissolved crystals was measured to identify the undissolved crystals as form $\mathrm{D}$, which proved that no crystal transformation occurred during the dissolution process. A clean beaker was weighed by an electronic balance (Analytical Balance, AL104, Mettler-Toledo) and the mass of it was recorded as $m_{0}$. The filtrate was filtered through a syringe filter $(0.22 \mu \mathrm{m})$ (Tianjin Legg Technology Co., Ltd, Tianjin, China) into the beaker and the total mass of the beaker and the filtrate was weighed again and recorded as $m_{1}$. The filtrate was dried under vacuum at $373.15 \mathrm{~K}$ and weighed at intervals. When the mass remained unchanged twice, the solvent could be considered to be completely evaporated and the final mass was recorded as $m_{2}$. The molar solubility of form D can be calculated by equation (2). Each experiment was performed three times to eliminate the error, and the final solubility was taken as the average of the three experimental results.

$$
\begin{aligned}
x= & \left\{\frac{\left[\left(1-V_{\mathrm{w}}\right) \rho_{\mathrm{d}}+V_{\mathrm{w}} \rho_{\mathrm{w}}\right]\left(m_{2}-m_{0}\right)}{M_{\mathrm{LM}}\left(m_{1}-m_{2}\right)}\right\} \\
& \times\left\{\frac{\left(1-V_{\mathrm{w}}\right) \rho_{\mathrm{d}}}{M_{\mathrm{d}}}+\frac{V_{\mathrm{w}} \rho_{\mathrm{w}}}{M_{\mathrm{w}}} \times \frac{\left[\left(1-V_{\mathrm{w}}\right) \rho_{\mathrm{d}}+V_{\mathrm{w}} \rho_{\mathrm{w}}\right]\left(m_{2}-m_{0}\right)}{M_{\mathrm{LM}}\left(m_{1}-m_{2}\right)}\right\}^{-1},
\end{aligned}
$$

where $x$ is the molar fraction solubility; $\rho_{\mathrm{d}}$ and $\rho_{\mathrm{w}}$ represent the density of DMSO and water at specific temperature respectively, with unit of $\mathrm{g} \mathrm{ml}^{-1} ; M_{\mathrm{d}}, M_{\mathrm{w}}$, and $M_{\mathrm{LM}}$ are the molar masses of DMSO, water and LM respectively, with unit of $\mathrm{g} \mathrm{ml}^{-1} ; V_{\mathrm{w}}$ is the volume fraction of water in DMSO-water mixed solvent. The values of parameters in equation (2) are shown in Table 2.

Since DMSO solvate will transform to form D, it is difficult to measure its solubility by conventional gravimetric methods. In the process of solvent-mediated polymorphic transformation (SMPT) from metastable form to stable form, the concentration of the solution will undergo three stages of change (Du et al., 2014), as shown in Fig. 2. During the dissolution stage of the metastable form, the solution concentration will rise from zero to the solubility of the metastable form and remain constant. When the stable form starts to nucleate and grow, the solute consumed by the nucleation and growth of stable form will be balanced by the dissolution of the metastable form. Thus, the concentration of the solution will be maintained at the solubility of the metastable form if the crystallization process is controlled by the nucleation and growth of the stable form. However, the concentration of the solution will drop to the solubility of the stable form and remain constant when the metastable form in solution is completely dissolved or the process of crystal transformation is controlled by the dissolution of the metastable form.

The solubility of LM DMSO solvate was measured by using real time Raman spectroscopy. Excess DMSO solvate was dissolved in a mixed solvent of DMSO $(15 \mathrm{ml})$ and water $(5 \mathrm{ml})$ to saturate the solution, while the temperature was 
controlled by a thermostatic water bath. Raman spectroscopy was used to collect in situ the Raman spectra of the solution in one minute intervals. When the characteristic peak intensity of DMSO solvate decreased, the experiment was immediately stopped. The suspension was filtered to obtain undissolved crystals and the PXRD data were measured to identify the undissolved crystals. The filtrate was filtered through a syringe filter and dried under vacuum at $373.15 \mathrm{~K}$. The solubility of DMSO solvate can be calculated by the equation (2).

The non-random two liquids (NRTL) equation is an activity coefficient model based on the concept of local composition and it can be used to calculate the activity of substances in the system. The NRTL equation in the ternary system can be simplified as:

$$
\begin{aligned}
\ln \gamma_{i}= & \frac{\left(G_{j i} x_{j}+G_{k i} x_{k}\right)\left(\tau_{j i} G_{j i} x_{j}+\tau_{k i} G_{k i} x_{k}\right)}{\left(x_{i}+G_{j i} x_{j}+G_{k i} x_{k}\right)^{2}} \\
& +\frac{\left[\tau_{i j} G_{i j} x_{j}^{2}+G_{i j} G_{k j} x_{i} x_{k}\left(\tau_{i j}-\tau_{k j}\right)\right]}{\left(x_{j}+G_{i j} x_{i}+G_{k j} x_{k}\right)^{2}} \\
& +\frac{\left[\tau_{i k} G_{i k} x_{k}^{2}+G_{i k} G_{j k} x_{j} x_{k}\left(\tau_{i k}-\tau_{j k}\right)\right]}{\left(x_{k}+G_{i k} x_{i}+G_{j k} x_{k}\right)^{2}} .
\end{aligned}
$$

$G_{i j}$ and $\tau_{i j}$ can be calculated by equations (4) and (5).

$$
\begin{gathered}
G_{i j}=\exp \left(-\alpha_{j i} \tau_{j i}\right), \\
\tau_{j i}=\left(g_{j i}-g_{i i}\right) / R T,
\end{gathered}
$$

where $g_{j i}-g_{i i}$ is the basic parameter of the NRTL equation which denotes the cross interaction energy. The parameter $\alpha_{j i}$ is related to the non-randomness in the mixture. The value of $\alpha_{j i}$ must be greater than 0 and less than 1 . Parameter $\alpha_{j i}$ in the ternary system follows equation (6)

$$
\alpha_{j i}=\alpha_{i j}
$$

2.6. Real-time monitoring of the SMDT process from LM DMSO solvate to form D using Raman spectroscopy

The SMDT process from LM DMSO solvate to form D was in situ monitored using Raman spectroscopy and the corresponding rate control step was ascertained. Moreover, the influences of water activity and temperature on the transformation process were also investigated. The effect of water activity on the SMDT process was investigated at $293.15 \mathrm{~K}$, in which $V_{\mathrm{w}}$ varied between 0 and 0.300 with a step size of 0.025 because of the impossibility of obtaining products when $V_{\mathrm{w}}$ is higher than 0.300. The effect of temperature on the SMDT process was conducted at $V_{\mathrm{w}}=0.250$ in the temperature range of $293.15-323.15 \mathrm{~K}$ with the step of $10 \mathrm{~K}$. At each temperature and $V_{\mathrm{w}}$, DMSO solvate $(2.0 \mathrm{~g})$ was added to DMSO-water mixed solvent $(3: 1 \mathrm{v} / \mathrm{v}, 40 \mathrm{ml})$ in a $100 \mathrm{ml}$ crystallizer and the stirring rate was controlled to be $300 \mathrm{rpm}$ with an overhead agitator. In all experiments, the systems were in situ monitored by Raman spectroscopy while the concentration of the solution was measured at certain intervals. The induction time was defined as the time from the starting of the experiment to the sudden drop of the Raman characteristic peak intensity of DMSO solvate, while the transformation time was defined as the time when the Raman characteristic peak intensity of DMSO solvate dropped to a constant value.

\section{Results and discussion}

\subsection{Characterization of DMSO solvate and form D of LM}

The PXRD diffraction patterns of LM form A, form C, form DMSO-2, DMSO solvate and form $\mathrm{D}$ are shown in supporting information (Fig. S1). The PXRD diffraction patterns of other reported solid forms of LM (Matsushima et al., 2005; Sardone et al., 2018) are listed in Fig. S2. It can be found that the PXRD data of DMSO solvate and form $\mathrm{D}$ are different from reported forms of LM. DMSO solvate has characteristic peaks at $2 \theta=$ $6.9 \pm, \quad 9.0 \pm, \quad 13.2 \pm, \quad 19.5 \pm, 24.0 \pm, 25.6 \pm, 27.2 \pm$, $31.6 \pm 0.2^{\circ}$ while form $\mathrm{D}$ has characteristic peaks at $2 \theta=$ $4.1 \pm 0.2^{\circ}, \quad 8.1 \pm 0.2^{\circ}, \quad 10.2 \pm 0.2^{\circ}, \quad 12.3 \pm 0.2^{\circ}, \quad 16.5 \pm 0.2^{\circ}$, $26.7 \pm 0.2^{\circ}, 29.0 \pm 0.2^{\circ}$.

Polarized light microscopy (PLM) was used to observe the morphology of DMSO solvate and form D of LM and the results are shown in supporting information (Fig. S3). DMSO solvate is in the shape of hexagonal flake while form D is needlelike. The TGA thermogram of DMSO solvate (Fig. S4) shows $12.71 \%$ weight loss at $433.15 \mathrm{~K}$, which is consistent with the theoretical DMSO content in DMSO solvate of LM with a stoichiometric ratio of 1:1 (about $13.00 \%$ ). form $\mathrm{D}$ shows no lose weight before decomposition, proving that it is an unsolvated form. The DSC curve of DMSO solvate (Fig. S5) shows an endothermic peak at $433.15 \mathrm{~K}$, which corresponds to the position of weight loss in the TGA curve, indicating that it is a desolvation peak. Another endothermic peak at $503.15 \mathrm{~K}$ indicates that crystals formed after desolvation of DMSO solvate melts. The DSC curve of form D shows an endothermic

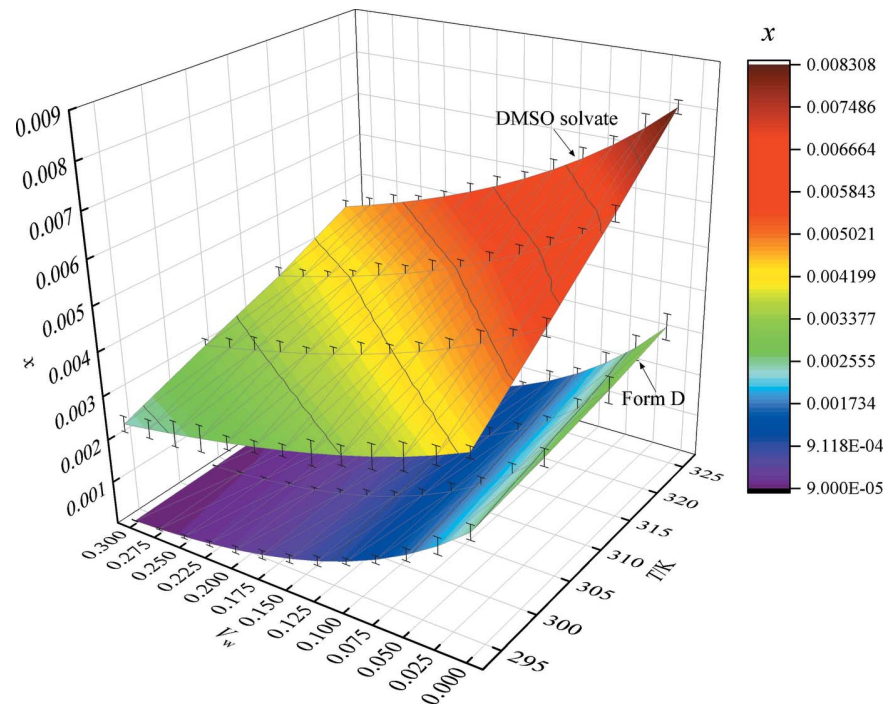

Figure 3

Solubility data of DMSO solvate and form D of LM in DMSO-water mixed solutions. 
peak at $473.15 \mathrm{~K}$, which should be the melting temperature of form $\mathrm{D}$.

Raman spectroscopy can be used to identify different polymorphs and can manifest the differences of their structures (Elbagerma et al., 2010). The Raman spectra results of DMSO solvate and form D of LM are shown in Fig. S6. Peaks at $1395 \mathrm{~cm}^{-1}$ and $1373 \mathrm{~cm}^{-1}$ were selected as characteristic peaks for DMSO solvate and form D, respectively and these peaks will be used to characterize their crystal transformation behavior in this work.

\subsection{Solubility and thermodynamic driving force for SMDT process}

In the crystal transformation process, solubility data can be used to evaluate the relative stability of different forms and to investigate the thermodynamic mechanism of the transformation (Zong et al., 2017; Du et al., 2014; Jouyban, 2008). In this study, the solubility data of LM DMSO solvate and form $\mathrm{D}$ in the mixed solvents of DMSO and water were measured, and the results are shown in Fig. 3. Specific data are given in Table S1.

It can be comprehended from Fig. 3 that the solubility of LM DMSO solvate and form D increased with the decreasing of $V_{\mathrm{w}}$ and increasing of temperature. The solubility surfaces of DMSO solvate and form D do not intersect and the solubility surface of DMSO solvate is permanently above the solubility surface of form D under the investigated conditions, indicating that the solubility of DMSO solvate is always higher than that of form D under the investigated conditions. According to the solubility data, form $\mathrm{D}$ is the stable form in the investigated temperature range, which implies that DMSO solvate should have a tendency to transform to form D in DMSO-water mixed solvent, regardless of the volume fraction of water. Furthermore, the NRTL equation was used to calculate the activities of DMSO solvate/form D, water and DMSO in

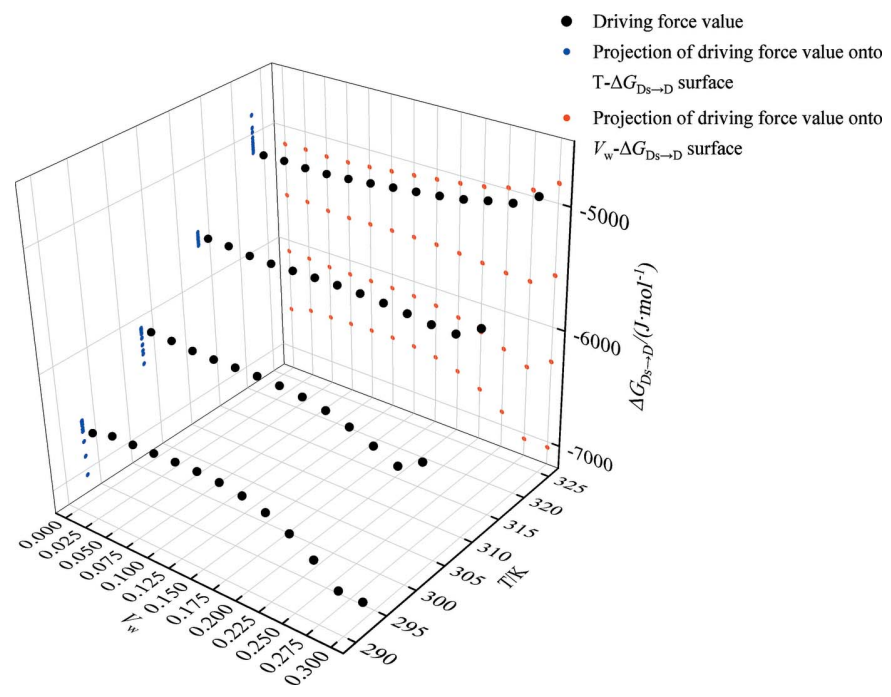

Figure 4

The thermodynamic driving force of SMDT from DMSO solvate to form D. saturated solution systems. The results of activity are listed in Table S1.

The thermodynamic driving force of the SMDT process from DMSO solvate to form D can be described by the Gibbs free energy difference $\left(\Delta G_{\mathrm{Ds} \rightarrow \mathrm{D}}\right)$ of DMSO solvate and form $\mathrm{D}$, which can be calculated by equation (7):

$$
\Delta G_{\mathrm{Ds} \rightarrow \mathrm{D}}=R T \ln \frac{f_{\mathrm{D}}}{f_{\mathrm{Ds}}}=R T \ln \frac{a_{\mathrm{D}}}{a_{\mathrm{Ds}}},
$$

where $R$ is the ideal gas constant, $8.314 \mathrm{~J} \mathrm{~mol}^{-1} \mathrm{~K}^{-1} ; T$ is the temperature with unit of $\mathrm{K}, f$ is the fugacity in units of $\mathrm{Pa}$ and $a$ is the activity.

The relationship among the driving force of the SMDT process, the temperature and $V_{\mathrm{w}}$ is displayed in a 3D map (Fig. 4). The values of $\Delta G_{\mathrm{Ds} \rightarrow \mathrm{D}}$ fluctuate within a smaller range with the increase of $V_{\mathrm{w}}$, indicating that the change of $V_{\mathrm{w}}$ have little effect on thermodynamic driving forces. In the temperature range of 293.15-323.15 K, the absolute values of $\Delta G_{\mathrm{Ds} \rightarrow \mathrm{D}}$ decrease with the increase of temperature, indicating that the driving force of the SMDT process diminishes as temperature increases. All values of $\Delta G_{\mathrm{Ds} \rightarrow \mathrm{D}}$ are negative, indicating that the SMDT process from DMSO solvate to form $\mathrm{D}$ should be spontaneous under the investigated conditions.

\subsection{SMDT process in situ monitored using Raman spectro-} scopy and determination of rate-controlling step

Raman spectroscopy was used to monitor in situ the SMDT process from LM DMSO solvate to form D. The suspension was taken out at fixed intervals during the SMDT process to analyze the liquid concentration and solid phase composition by gravimetric method and PXRD calibration curve, respectively. The representative results at $T=293.15 \mathrm{~K}$ and $V_{\mathrm{w}}=$ 0.250 are graphically shown in Fig. 5 and part of the PXRD patterns are shown in Fig. S8.

It can be seen from Fig. 5 that the trend of the mass fraction of DMSO solvate in the suspended solid phase measured by PXRD data coincide with the trend represented by the relative Raman intensity of the characteristic peak of DMSO

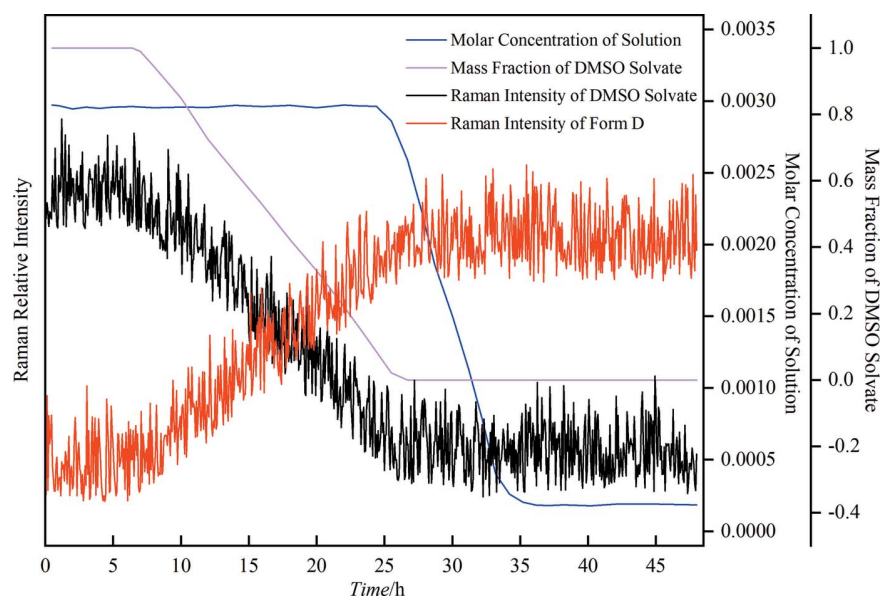

Figure 5

The SMDT process from DMSO solvate to form D in mixed solvent of DMSO and water $\left(V_{\mathrm{w}}=0.250\right)$ at $293.15 \mathrm{~K}$. 
solvate $\left(1395 \mathrm{~cm}^{-1}\right)$, manifesting that the real time Raman data could accurately represent the solid state composition of the SMDT process. Fig. S8 shows the change of suspended solid phase over time more intuitively. During the first $6.4 \mathrm{~h}$ of transformation process, the relative Raman intensity of the characteristic peak of DMSO solvate kept constant, which indicated that no transformation phenomenon occurred. The characteristic peak of form D $\left(1373 \mathrm{~cm}^{-1}\right)$ emerged at about $6.4 \mathrm{~h}$, indicating the end of the induction process and the beginning of the nucleation and growth process of form $\mathrm{D}$. Then, the relative Raman intensity of form D gradually increased while the relative Raman intensity of DMSO solvate decreased, revealing the proceeding of the transformation process. At approximately $26.7 \mathrm{~h}$, the characteristic peak of DMSO solvate thoroughly vanished and the relative Raman intensity of form D reached maximum and remained unchanged, indicating that DMSO solvate had entirely transformed into form $\mathrm{D}$, corresponding to the end of the SMDT process. Moreover, the liquid phase concentration curve shows that the solution concentration stayed at a plateau for $2.3 \mathrm{~h}$ before the transformation process was finished, indicating that the consuming rate of supersaturation by the growth of stable form was lower than the generating rate of supersaturation by the dissolution of metastable form (O'Mahony et al., 2012). Then, the concentration gradually decayed to the saturation concentration of form $\mathrm{D}$ within about $11.8 \mathrm{~h}$, and whereafter, remained unchanged. Moreover, the system underwent a long induction time (about $6.4 \mathrm{~h}$ ) before the transformation process started, which may be caused by the comparative difficulty to form the crystal nuclei of stable form $\mathrm{D}$. According to the four instances described by O'Mahony et al. (2012), the SMDT process from LM DMSO solvate to form $\mathrm{D}$ is controlled by the nucleation and growth of form D.

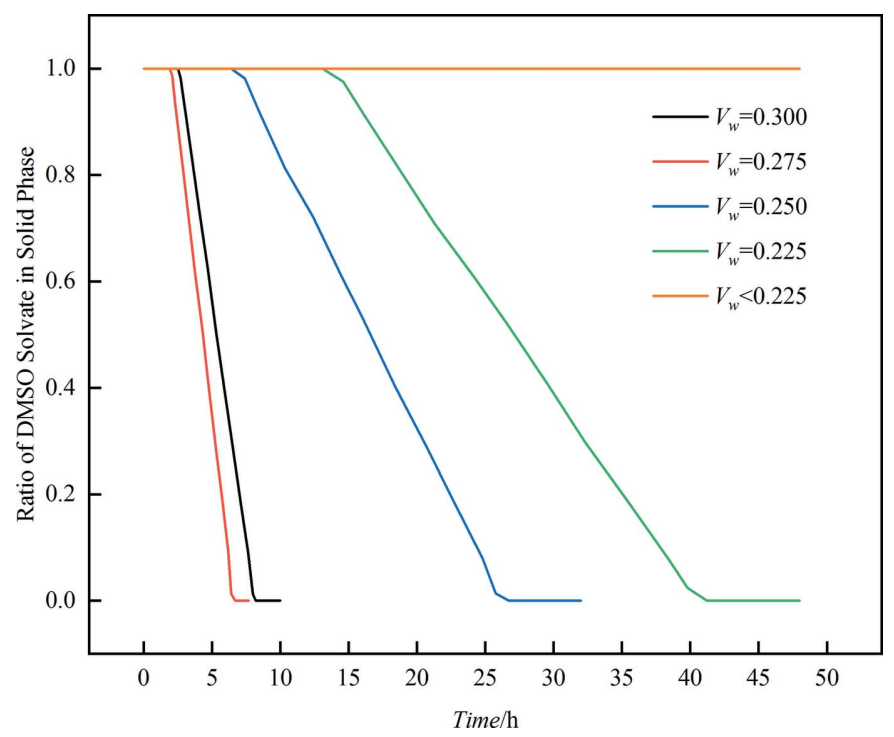

Figure 6

Effect of $V_{\mathrm{w}}$ on the SMDT process from LM DMSO solvate to form D at $293.15 \mathrm{~K}$.
Table 3

Induction time $\left(t_{\text {ind }}\right)$ and transformation time $\left(t_{\text {tra }}\right)$ of LM DMSO solvate to form D in DMSO-water mixed solvent with different $V_{\mathrm{w}}$.

\begin{tabular}{lllll}
\hline & $V_{\mathrm{w}}$ & & & \\
\cline { 2 - 5 } & 0.225 & 0.250 & 0.275 & 0.300 \\
\hline$t_{\text {ind }}(\mathrm{h})$ & 13.1 & 6.4 & 1.9 & 2.5 \\
$t_{\text {tra }}(\mathrm{h})$ & 41.2 & 26.7 & 6.7 & 8.2 \\
\hline
\end{tabular}

\subsection{Effect of water activity on the SMDT process}

In this work, the effect of water activity on the SMDT process in the DMSO-water mixed solvent was investigated and the results are shown in Fig. 6 and Table 3 .

It can be seen from Fig. 6 that the solvent molecules in DMSO solvate could be removed only when $V_{\mathrm{w}}$ is higher than or equal to 0.225 . However, it is inconsistent with the conclusion from the thermodynamic driving force discussed in Section 3.2 that the SMDT process is spontaneous regardless of the $V_{\mathrm{w}}$. The contradiction demonstrates that the SMDT process probably follows a special mechanism which is not only controlled by the thermodynamic driving force. The activity of water might play a vital role in this process. It can be deduced from the experimental phenomenon and activity data that the necessary condition for the SMDT process is that the activity of water must be higher than the activity of DMSO. Based on these data, a new mechanism for the SMDT process of LM is proposed.

The SMDT process from LM DMSO solvate to form D may be divided into four steps. Since the specific structure of lenvatinib mesylate was not successfully determined, the term 'LM' is used here to refer to the co-crystal/salt structure formed by one molecule of lenvatinib and one molecule of methanesulfonic acid. Firstly, when DMSO solvate is dissolved in the mixed solvent, the intermolecular interaction (probably hydrogen bond) between LMs and DMSO molecules will not be broken straightaway. In other words, the structure of LM.DMSO could be maintained in the solution for some time. Secondly, water molecules might be promoted to replace DMSO molecules in the original structure after a period of induction time if the activity of water is higher than the activity of DMSO, which would lead to the formation of intermediates combined by the intermolecular interactions (probably by hydrogen bonds) of LMs and water molecules. Thirdly, water

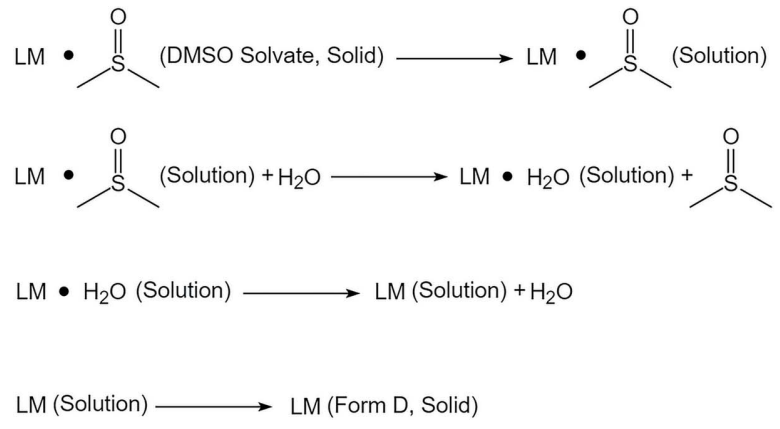

Figure 7

The mechanism of the SMDT process from LM DMSO solvate to form D. 
molecules in the intermediate molecular structure could also be removed, resulting in the freeing of LMs. Finally, isolated LMs might form new structures by solute-solute intermolecular interactions, which can lead to the start of the nucleation process of form $\mathrm{D}$. The whole SMDT mechanism is demonstrated in Fig. 7. Overall, water might play the role of catalyst throughout the desolvation process. Thus, kinetically, when the activity of water is lower than the activity of DMSO, the impossibility of triggering the second step will inhibit the SMDT process, even if the desolvation of DMSO solvate is a thermodynamic spontaneous process.

In order to verify this mechanism, an extra cooling crystallization experiment was designed. Appropriate amount of LM DMSO solvate was added to a DMSO-water mixed solvent with $V_{\mathrm{w}}=0.250$ at $323.15 \mathrm{~K}$ to form a saturated solution. The saturated solution was cooled to $275.15 \mathrm{~K}$ at a cooling rate of $0.5 \mathrm{~K} \mathrm{~min}^{-1}$, and crystals were found to precipitate. After the cooling was completed, stirring was continued for $12 \mathrm{~h}$ at the constant temperature. After filtering and vacuum drying, the crystal product was taken for XRD characterization and it was found that the hydrated crystalline form DMSO-2 was obtained (Sardone et al., 2018). TGA and DSC curve (shown in Figs. S10 and S11) verified that DMSO-2 is a tetrahydrate (the weight loss detected by TGA is $12.07 \%$ ) and its desolvation temperature is about $379.25 \mathrm{~K}$. The results of this cooling crystallization are consistent with the SMDT mechanism mentioned above. The rapid decreasing of temperature would promptly elevate the supersaturation of the system and significantly reduce the nucleation time, which would make LM. $\mathrm{H}_{2} \mathrm{O}$ have no enough time to remove water molecules from the structure. Generally, lower temperature will favor the formation of intermolecular hydrogen bonds (Kim et al., 2020). Consequently, the tetrahydrate crystal form DMSO-2 was formed. Moreover, the desolvation temperature of DMSO-2 (about $379.25 \mathrm{~K}$ ) is higher than the boiling point of water $(373.15 \mathrm{~K})$, revealing that water molecules participate

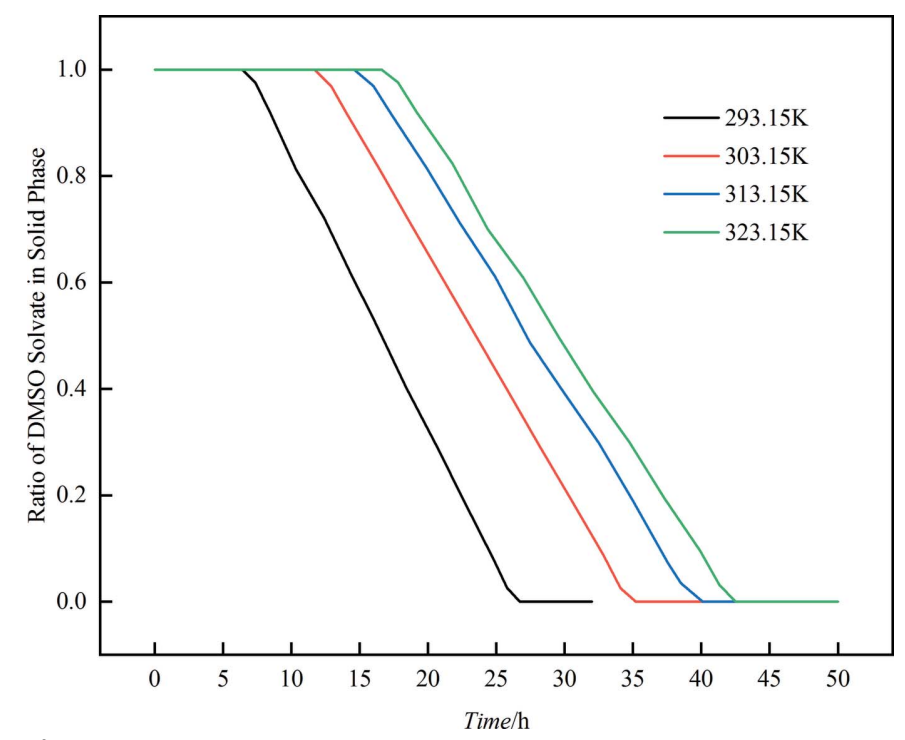

Figure 8

Effect of temperature on the SMDT process from LM DMSO solvate to form D in mixed solvent of DMSO and water $\left(V_{\mathrm{w}}=0.250\right)$.
Table 4

Induction time and transformation time of LM DMSO solvate to form D at different temperatures.

\begin{tabular}{lllll}
\hline & $T(\mathrm{~K})$ & & & \\
\cline { 2 - 5 } & 293.15 & 303.15 & 313.15 & 323.15 \\
\hline$t_{\text {ind }}(\mathrm{h})$ & 6.4 & 11.7 & 14.8 & 16.6 \\
$t_{\text {tra }}(\mathrm{h})$ & 26.7 & 35.2 & 40.1 & 42.5 \\
\hline
\end{tabular}

in the formation of the lattice structure and were tightly bonded with LM through intermolecular interactions (Fátima Pina et al., 2012; Chen et al., 2008). Moreover, the desolvation energy of DMSO-2 is $10.2 \mathrm{~kJ} \mathrm{~mol}^{-1}$ according to the result of DSC, which is within the energy range of the corresponding intermolecular hydrogen bond $\left(5-40 \mathrm{~kJ} \mathrm{~mol}^{-1}\right.$ ) (Pauling, 1960), revealing that the intermolecular interaction in DMSO2 is hydrogen bond. Therefore, the thermal analysis results are also consistent with the SMDT mechanism proposed above.

It is also worth noting in Fig. 6 that, in general, the induction time and the transformation time of the SMDT process shortened significantly with the increase of $V_{\mathrm{w}}$ at the same temperature. The induction time and the transformation time of DMSO solvate at $V_{\mathrm{w}}=0.225$ were respectively $13.1 \mathrm{~h}$ and $41.2 \mathrm{~h}$ while they are $1.9 \mathrm{~h}$ and $6.7 \mathrm{~h}$ respectively when $V_{\mathrm{w}}=$ 0.275 . This is because relatively high water activity can promote the formation of hydrate intermediates, resulting in a shorter induction time and transformation time. However, when $V_{\mathrm{w}}=0.300$, the induction time and the transformation time of the SMDT process were both anomalously longer than those of $V_{\mathrm{w}}=0.275$. This may be due to the strong hydrogen bond acceptor (HBA) propensity and hydrogen bond donor (HBD) propensity of water (Gu et al., 2004), while LM have groups that have both HBA propensity and HBD propensity, such as its primary and secondary amide groups. Therefore, when water activity is higher, the tendency of forming a complex hydrogen bond network between LMs and water molecules enhances, resulting in more difficult removal of water molecules in the hydrate intermediate. Strong solutesolvent intermolecular interactions and low raw material concentration (because of the decreasing solubility of DMSO solvate as the water activity declined) will increase the difficulty of forming solute-solute intermolecular interactions, which will then increase the difficulty of nucleation of form $\mathrm{D}$. As a result, the induction time and the transformation time will be prolonged. In fact, the reason why the viscosity of the system skyrockets when $V_{\mathrm{w}}>0.300$ might be also due to this mechanism.

\subsection{Effect of temperature on the SMDT process}

The effect of temperature on the desolvation process was also studied and the results are shown in Fig. 8. Obviously, the induction time and the transformation time of the SMDT process in the mixed solvent of $V_{\mathrm{w}}=0.250$ became longer as the temperature increased. The induction time increased from $6.4 \mathrm{~h}$ at $293.15 \mathrm{~K}$ to $16.6 \mathrm{~h}$ at $323.15 \mathrm{~K}$, while the transforma- 
tion time increased from $26.7 \mathrm{~h}$ at $293.15 \mathrm{~K}$ to $42.5 \mathrm{~h}$ at $323.15 \mathrm{~K}$, as enumerated in Table 4.

There are two reasons for this abnormal result. Firstly, rising temperature will result in a lower thermodynamic driving force, which will lead to an increase in induction time and transformation time. Secondly, in the second step of the above four-step SMDT mechanism, the hydrogen bond between LMs and DMSO molecules in the solution would be broken while new hydrogen bond between LMs and water molecules will be formed. The higher temperature is not favorable for the formation of the new hydrogen bonds, which will affect the formation of crystal nuclei of form $\mathrm{D}$. Thus, the transformation speed will gear down.

\section{Conclusions}

Two new solid forms of LM were obtained and characterized by PXRD, TGA, DSC, PLM and Raman spectroscopy for the first time. To investigate the transformation behaviors from DMSO solvate to form $\mathrm{D}$, the PXRD calibration curve was established to quantify the mass fraction of the two solid forms in a mixture. The solubility data of DMSO solvate and form D in DMSO-water mixed solvents were measured and correlated using NRTL equation. The solubility data were used to evaluate the thermodynamic driving force of the SMDT process from DMSO solvate to form D. It was found that a turning point at $V_{\mathrm{w}}=0.225$ existed. When $V_{\mathrm{w}}$ was relatively high, DMSO solvate would desolvate and transform to form D while the desolvation transformation process would not take place at lower $V_{\mathrm{w}}$. Through further investigations, it was found that the activity of water was the most important parameter that determined whether or not the SMDT process could happen. Moreover, Raman and solution concentration data displayed that the SMDT process was controlled by nucleation and growth of form D. Furthermore, the affecting mechanism of water activity and temperature on the SMDT process were investigated. The results demonstrated that the increase in temperature slowed down the SMDT process because of the decreasing thermodynamic driving force and the obstruction of forming new hydrogen bonds between LMs and water molecules. One new SMDT mechanism was suggested and discussed according to the experimental results and the mechanism was verified by cooling crystallization experiments.

\section{Funding information}

The following funding is acknowledged: National Natural Science Foundation of China (grant No. 21978201; grant No. 21908159).

\section{References}

Anwar, J., Tarling, S. E. \& Barnes, P. (1989). J. Pharm. Sci. 78, 337342.

Apperley, D. C., Fletton, R. A., Harris, R. K., Lancaster, R. W., Tavener, S. \& Threlfall, T. L. (1999). J. Pharm. Sci. 88, 12751280.

Barbas, R., Font-Bardia, M. \& Prohens, R. (2018). Cryst. Growth Des. 18, 3740-3746.
Bērziņš, A., Trimdale, A., Kons, A. \& Zvaniņa, D. (2017). Cryst. Growth Des. 17, 5712-5724.

Bingham, A. L., Hughes, D. S., Hursthouse, M. B., Lancaster, R. W., Tavener, S. \& Threlfall, T. L. (2001). Chem. Commun. pp. 603-604.

Black, S. N., Cuthbert, M. W., Roberts, R. J. \& Stensland, B. (2004). Cryst. Growth Des. 4, 539-544.

Bond, A. D. (2009). Curr. Opin. Solid State Mater. Sci. 13, 91-97.

Braun, D. E., Gelbrich, T., Wurst, K. \& Griesser, U. J. (2016). Cryst. Growth Des. 16, 3480-3496.

Brittain, H. G. (2009). Drugs and the Pharmaceutical Science, Vol. 192, pp. 1-24. Florida: CRC Press.

Chen, J. X., Wang, J. K., Ulrich, J., Yin, Q. X. \& Xue, L. Z. (2008). Cryst. Growth Des. 8, 1490-1494.

Croker, D. M., Hennigan, M. C., Maher, A., Hu, Y., Ryder, A. G. \& Hodnett, B. K. (2012). J. Pharm. Biomed. Anal. 63, 80-86.

Du, W., Yin, Q. X., Hao, H. X., Bao, Y., Zhang, X., Huang, J. T., Li, X., Xie, C. \& Gong, J. B. (2014). Ind. Eng. Chem. Res. 53, 56525659.

Elbagerma, M. A., Edwards, H. G. M., Munshi, T., Hargreaves, M. D., Matousek, P. \& Scowen, I. J. (2010). Cryst. Growth Des. 10, 23602371.

Gu, C. H., Li, H., Gandhi, R. B. \& Raghavan, K. (2004). Int. J. Pharm. 283, 117-125.

Gu, C. H., Young, V. Jr \& Grant, D. J. W. (2001). J. Pharm. Sci. 90, 1878-1890.

Guo, N. N., Hou, B. H., Wang, N., Xiao, Y., Huang, J. J., Guo, Y. M., Zong, S. Y. \& Hao, H. X. (2018). J. Pharm. Sci. 107, 344-352.

Hao, H. X., Barrett, M., Hu, Y., Su, W. Y., Ferguson, S., Wood, B. \& Glennon, B. (2012). Org. Process Res. Dev. 16, 35-41.

Hao, H. X., Su, W. Y., Barrett, M., Caron, V., Healy, A.-M. \& Glennon, B. (2010). Org. Process Res. Dev. 14, 1209-1214.

Jiang, C., Wang, Y. L., Yan, J. Q., Yang, J. X., Xiao, L. P. \& Hao, H. X. (2015). Org. Process Res. Dev. 19, 1752-1759.

Jiang, S. F., Jansens, P. J. \& ter Horst, J. H. (2010). Cryst. Growth Des. 10, 2541-2547.

Jouyban, A. (2008). J. Pharm. Pharm. Sci. 11, 32-58.

Kim, S.-H., Ju, K.-S., Ri, H.-H. \& Ri, S.-P. (2020). Chem. Phys. Lett. 742, 137146.

Li, Y., Chow, P. S. \& Tan, R. B. H. (2011). Int. J. Pharm. 415, 110 118.

Llinàs, A. \& Goodman, J. M. (2008). Drug Discovery Today, 13, 198210.

Maini, L., Braga, D., Farinella, F., Melotto, E., Verzini, M., Brescello, R., Michieletto, I. \& Munari, I. (2018). Cryst. Growth Des. 18, 37743780 .

Mangin, D., Puel, F. \& Veesler, S. (2009). Org. Process Res. Dev. 13, 1241-1253.

Matsushima, T., Nakamura, T., Yoshizawa, K., Kamada, A., Ayata, Y., Suzuki, N., Arimoto, I., Sakaguchi, T., Gotoda, M., Nakamura, H., Kamata, A., Arimoto, T., Atsushi, K., Iwasaki, S., Kazuhiro, Y., Masaharu, G., Taiju, N., Takahisa, S., Tatsu, A., Tomohiro, M., Yusuke, A., Suzuki, N. A. I., Naoko, S., Itaru, A., Nakamura, P. \& Arimoto, N. (2005). Google Patents. Patent number WO 2005063713 A1.

Okamoto, K., Ikemori-Kawada, M., Jestel, A., von König, K., Funahashi, Y., Matsushima, T., Tsuruoka, A., Inoue, A. \& Matsui, J. (2015). ACS Med. Chem. Lett. 6, 89-94.

O'Mahony, M. A., Maher, A., Croker, D. M., Rasmuson, C. \& Hodnett, B. K. (2012). Cryst. Growth Des. 12, 1925-1932.

Ouyang, J. B., Wang, J. K., Huang, X., Gao, Y., Bao, Y., Wang, Y. L., Yin, Q. X. \& Hao, H. X. (2014). Ind. Eng. Chem. Res. 53, 1685916863.

Pauling, L. (1960). The Nature of the Chemical Bond. New York: Cornell University Press.

Pina, M. F., Pinto, J. F., Sousa, J. J., Fábián, L., Zhao, M. \& Craig, D. Q. M. (2012). Mol. Pharm. 9, 3515-3525.

Qiu, J.-B., Li, G., Sheng, Y. \& Zhu, M.-R. (2015). J. Pharm. Biomed. Anal. 107, 298-303. 
Sardone, N., Giaffreda, S. L., Gambini, A., Petrolati, A., Allegrini, P. \& Modena, E. (2018). Google Patents. Patent number EP 3299360 A1.

Schmidt, A. C., Niederwanger, V. \& Griesser, U. J. (2004). J. Therm. Anal. Calorim. 77, 639-652.

Schöll, J., Bonalumi, D., Vicum, L., Mazzotti, M. \& Müller, M. (2006). Cryst. Growth Des. 6, 881-891.

Shi, P., Xu, S. J., Du, S. C., Rohani, S., Liu, S. Y., Tang, W. W., Jia, L. N., Wang, J. K. \& Gong, J. B. (2018). Cryst. Growth Des. 18, 59475956.
Shi, Z. C., Wang, Z. Z., Zhang, T., Dang, L. P. \& Wei, H. Y. (2015). RSC Adv. 5, 98050-98056.

Wirth, D. D. \& Stephenson, G. A. (1997). Org. Process Res. Dev. 1, 55-60.

Yang, L. Q., Hao, H. X., Zhou, L. N., Chen, W., Hou, B. H., Xie, C. \& Yin, Q. X. (2013). Ind. Eng. Chem. Res. 52, 17667-17675.

Yang, X. C., Sarma, B. \& Myerson, A. S. (2012). Cryst. Growth Des. 12, 5521-5528.

Zong, S. Y., Wang, J. K., Xiao, Y., Wu, H., Zhou, Y. N., Guo, Y. M., Huang, X. \& Hao, H. X. (2017). J. Mol. Liq. 241, 399-406. 